\title{
Broadband enhancement of spontaneous emission in a photonic-plasmonic structure
}

Zhu, Xiaolong; Xie, Fengxian; Shi, Lei; Liu, Xiaohan; Mortensen, N. Asger; Xiao, Sanshui ; Zi, Jian; Choy, Wallace

Published in:
Optics Letters

Link to article, DOI:

10.1364/OL.37.002037

Publication date:

2012

Document Version

Publisher's PDF, also known as Version of record

Link back to DTU Orbit

Citation (APA):

Zhu, X., Xie, F., Shi, L., Liu, X., Mortensen, N. A., Xiao, S., Zi, J., \& Choy, W. (2012). Broadband enhancement of spontaneous emission in a photonic-plasmonic structure. Optics Letters, 37(11), 2037-2039.

https://doi.org/10.1364/OL.37.002037

\section{General rights}

Copyright and moral rights for the publications made accessible in the public portal are retained by the authors and/or other copyright owners and it is a condition of accessing publications that users recognise and abide by the legal requirements associated with these rights.

- Users may download and print one copy of any publication from the public portal for the purpose of private study or research.

- You may not further distribute the material or use it for any profit-making activity or commercial gain

- You may freely distribute the URL identifying the publication in the public portal 


\title{
Broadband enhancement of spontaneous emission in a photonic-plasmonic structure
}

\author{
Xiaolong Zhu, ${ }^{1,2,3}$ Fengxian Xie, ${ }^{2}$ Lei Shi, ${ }^{1}$ Xiaohan Liu, ${ }^{1}$ N. Asger Mortensen, ${ }^{3}$ Sanshui Xiao, ${ }^{3}$ \\ Jian $\mathbf{Z i}^{1, * *}$ and Wallace Choy ${ }^{2,4}$ \\ ${ }^{1}$ Department of Physics, Key Laboratory of Micro and Nano Photonic Structures (Ministry of Education), and State Key Laboratory \\ of Surface Physics, Fudan University, Shanghai 200433, China \\ ${ }^{2}$ Department of Electrical and Electronic Engineering, The University of Hong Kong, Pokfulam Road, Hong Kong, China \\ ${ }^{3}$ Department of Photonics Engineering, Technical University of Denmark, DK-2800 Kgs. Lyngby, Denmark \\ ${ }^{4}$ e-mail: chchoy@eee.hku.hk \\ ${ }^{*}$ Corresponding author: jzi@fudan.edu.cn
}

Received February 28, 2012; revised March 23, 2012; accepted March 23, 2012; posted March 23, 2012 (Doc. ID 163809); published May 30, 2012

\begin{abstract}
We demonstrate that a broadband enhancement of spontaneous emission can be achieved within a photonicplasmonic structure. The structure can strongly modify the spontaneous emission by exciting plasmonic modes. Because of the excited plasmonic modes, an enhancement up to 30 times is observed, leading to a 4 times broader emission spectrum. The reflectance measurement and the finite-difference time-domain simulation are carried out to support these results. (C) 2012 Optical Society of America

OCIS codes: $240.6680,260.5740$.
\end{abstract}

Enhancing the spontaneous emission of light is at the heart of many photonic applications, ranging from lowthreshold lasing [1] and bright LEDs [2] to single-photon light sources [3]. In a suitably tailored dielectric environment that is structured at the scale of the wavelength, the spontaneous emission can be locally increased [4]. The enhancement of spontaneous emission rates in an optical resonant cavity, known as the Purcell effect, is described by the Purcell factor [ㅁ],$F_{p}=\frac{3}{4 \pi^{2}} \frac{Q}{V}\left(\frac{\lambda}{n}\right)^{3}$, where $\lambda / n$ is the wavelength within the material and $Q$ and $V$ are the quality factor and mode volume of the cavity, respectively.

Most work so far has focused on achieving high Purcell factors by optimizing $Q$ in low-loss dielectric optical cavities, leading to enhanced interaction between light and matter within a very narrow bandwidth [6] . An alternative way to increase the Purcell factor is to reduce the mode volume. Because of the oscillations of free electrons at metal interfaces, plasmonic nanostructures can concentrate optical fields into nanoscale volumes [7].

With modest $Q$ and extremely small mode volume in a designed plasmonic structure, a high Purcell factor can also be achieved [8], enabling broadband-enhanced light emission. However, surface-plasmon-improved emissions recently reported are mostly based on rough metallic films $[9,10]$ or one-dimensional grating structures $[9,11]$. In this Letter, by a simple and inexpensive method based on a self-assembly technology, we fabricate a two-dimensional (2D) plasmonic structure on a glass substrate, consisting of a gold-capped monolayer array of polystyrene (PS) spheres.

Fluorescent materials with host/guest system composed of 4,4'-bis(N-carbazolyl)-1,1'-biphenyl (CBP) and tris(1-phenylisoquinolinolato- $\mathrm{C}^{2}, \mathrm{~N}$ ) iridium(III) [Ir(piq) ${ }_{3}$ ] (1 wt. \% concentration) are deposited on the structure in order to study the spontaneous light emission. The composited system, a so-called hybrid photonic-plasmonic structure, supports plasmonic [surface plasmon polariton (SPP)] modes. We have measured photoluminescence (PL) spectra for the structured samples and have obtained a strong enhancement of spontaneous emission. Such enhancement is demonstrated by Purcell effect in these optical modes, supported by the reflectance measurement and the finite-difference timedomain (FDTD) simulation results.

Figure 1(a) schematically illustrates the fabrication process of the photonic-plasmonic structure with fluorescent bulk heterojunction (BHJ) materials. First, a monolayer hexagonal-close-packed array of monodisperse PS spheres with a diameter of $600 \mathrm{~nm}$ (size dispersion $1 \%$ ) was formed on a pretreated glass surface by a self-assembly method [12]. Second, a $50 \mathrm{~nm}$ thick gold film was deposited on the monolayer array of PS spheres by thermal evaporation. Because of the shadow effect, metallic caps can be formed on the PS spheres. After

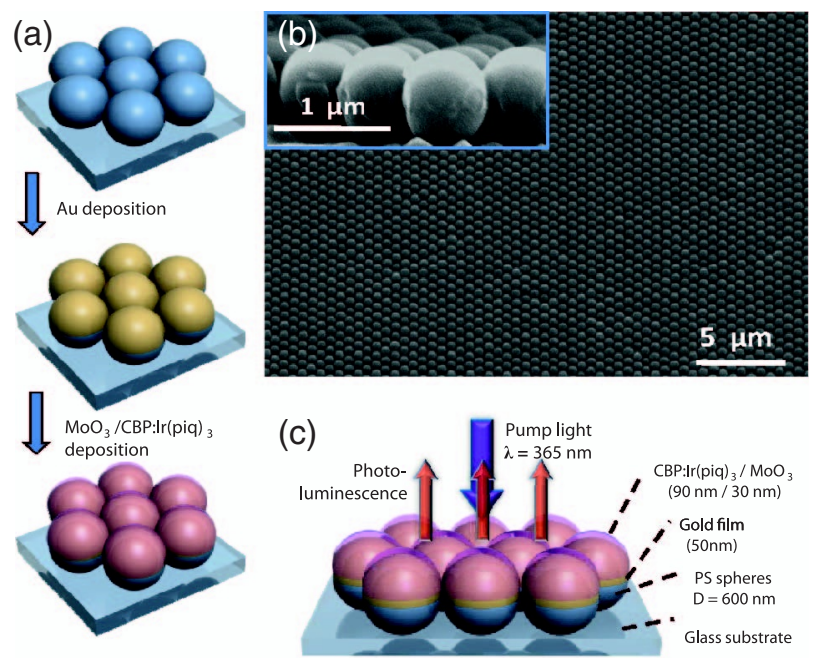

Fig. 1. (Color online) (a) Schematics of the self-assembly and deposition processes. (b) Top-down and cross-sectional (inset) scanning electron microscope (SEM) images of the accomplished hybrid photonic-plasmonic structures. (c) Schematic of a photonic-plasmonic structure functionalized with fluorescent BHJ material. 
that, a $30 \mathrm{~nm} \mathrm{MoO}_{3}$ barrier layer and a $90 \mathrm{~nm}$ organic fluorescent BHJ material CBP:Ir(piq) $)_{3}$ are thermally evaporated. Here the $\mathrm{MoO}_{3}$ layer is employed as an electron blocking layer to prohibit excitons in the organic semiconducting layer far from the metallic electrode, avoiding the quenching effect at the metal/organic interface [13].

Figure 1(b) illustrates top-down and cross-sectional (inset) SEM images of a portion of the accomplished hybrid structure. We find that these spherical elements are arranged regularly in a hexagonal lattice. A schematic of the combined structure with parameters is illustrated in Fig. 1(c).

In order to investigate the enhancement of light emission comparatively, we also manufacture reference samples with barrier and fluorescent layers on bare and gold coated plain glass substrates. It is worth mentioning that we select the blend of CBP:Ir(piq) ${ }_{3}$ with an emission peak around $630 \mathrm{~nm}$ [14], which is comparable with the period of the self-assembled optical structure.

PL and reflectance spectra of the samples were measured using a microspectrophotometer, which was connected to a Nikon Eclipse TE-2000-U fluorescence microscope equipped with a $20 \times$ objective, a narrow bandpass (365 nm line of mercury spectrum) fluorescence filter cube, and a high-power mercury lamp. PL emitted from the fluorescent BHJ material was pumped in a focused spot $(\sim 100 \mu \mathrm{m})$ confined by an aperture and then collected by the objective.

Figure 2 shows the emission spectra at normal incidence for the structured and reference samples together with a reflectance spectrum for the structured sample. We find that no obvious enhancement is observed for the sample with gold coated glass substrate as compared to the one with plain glass substrate. (A small increase in the luminescence intensity is shown as expected because the metal reflects pump light back through the fluorescent layer, doubling the effective path of the incident light.) But for the structured sample, multifold broadband enhancement takes place in the spectral range of interest. In general, there are two dips in the reflectance spectrum of the structured sample. They are attributed to

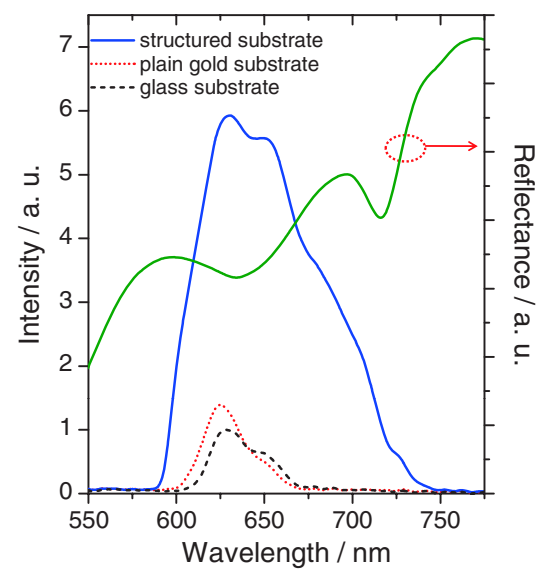

Fig. 2. (Color online) Normal-incidence reflection and emission spectra (solid lines) from the sample with photonicplasmonic structure. The emission spectra from the samples with bare (dashed line) and gold coated (dotted line) plain glass substrates are also plotted for comparison. Emission spectra are normalized to the one with plain glass substrate. two optical modes excited in the photonic-plasmonic structure, which will be explained later. For the mode at $\lambda=633 \mathrm{~nm}(Q \simeq 16)$, a 3 times enhancement in emission is observed when compared with that for the reference sample with the plain gold film. For the mode located at $\lambda=716 \mathrm{~nm}(Q \simeq 25)$, emission is enhanced up to 30 times over the region of the mode, and thus the FWHM of the emission spectrum is broadened from 25 to $95 \mathrm{~nm}$.

To explain the above phenomenon, a normal-incidence reflectance spectrum is obtained by three-dimensional (3D)-FDTD simulations. Figure 3(a) shows simulated and experimental reflection spectra for the hybrid photonic-plasmonic structure. Figure 3(b) presents the refractive indices of $\mathrm{MoO}_{3}$ and CBP:Ir(piq) $)_{3}$. There are two dips (G1 and G2) in the spectra in Fig. 3(a), corresponding to the modes of the light coupling to the system. This is because, for a $2 \mathrm{D}$ periodical lattice, if the in-plane component of incident light wave vector $K_{\|}$satisfies the following phase-matching condition [7],$K_{O R}=K_{\|}+G_{m n}$, then coupled optical resonances can be excited. Here $K_{O R}$ is the wave vector of the coupled optical resonance and $G_{m n}=2 \pi / \Lambda$ represents the reciprocal lattice vectors of the 2D lattice. By using the weak scattering approximation, $\left.\Lambda=\frac{\sqrt{3}}{2}(m \mathbf{a}+n \mathbf{b})\right)$, where $m$ and $n$ are integers and $\mathbf{a}$ and $\mathbf{b}$ are two basis lattice vectors in the 2D lattice. From Eq. (2), we find that the emission characteristics of our system are with the possibility of tuning via using the dispersion relation at oblique incidence [15] or changing the lattice structures by applying elastomeric substrates $[16,17]$.

In order to further explore the properties of each mode, we calculate the spatial distributions of their electric field. It should be pointed out that the contact points
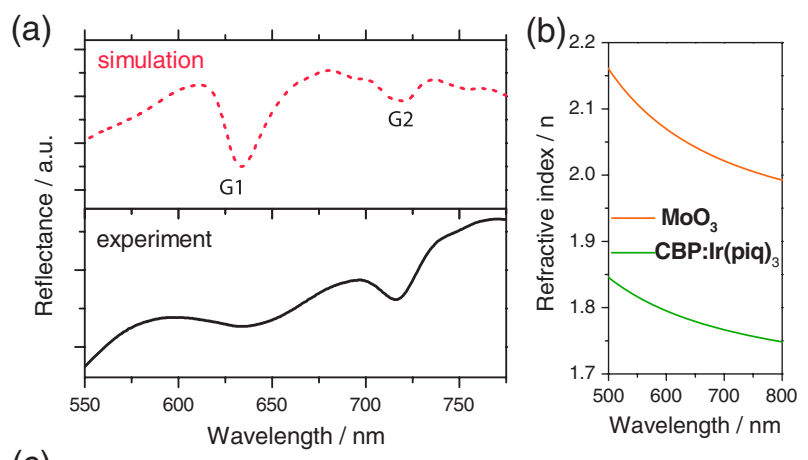

(c)

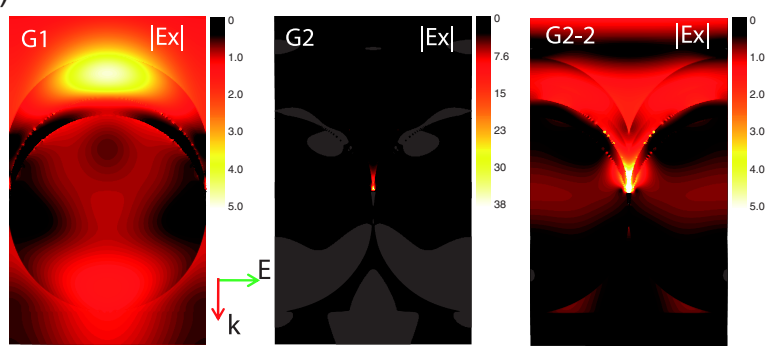

Fig. 3. (Color online) (a) Simulated (dashed line) and measured (solid line) normal-incidence reflection spectra of the photonic-plasmonic structure. (b) Refractive indices of the $\mathrm{MoO}_{3}$ and CBP:Ir(piq) $)_{3}$. (c) E-field distribution of selected modes as indicated in (a). 
of Au caps are made smooth in the simulations, in accordance with any practical realization; such that they do not form point contacts which would otherwise generate field singularities. Figure 3(c) shows the $\left|E_{x}\right|$ field crosssection profiles in the direction normal to the $2 \mathrm{D}$ periodicity. For the G1 mode at $\lambda=633 \mathrm{~nm}$, it has a more complex field distribution extending both into the metaldielectric interface and the fluorescent layer. This mode corresponds to a hybrid SPP mode at the gamma point, which is with a slow group velocity at its resonant frequency [9]. Small group velocities produce large densities of photonic states at a wide range of spectra. Eventually the plasmonic structures presented here lead to the enhanced spontaneous emission.

For the G2 mode at $\lambda=716 \mathrm{~nm}$, the electric field concentrates at the sharp corner formed by the adjacent metallic semishells, which is believed to be a SPP mode. In order to further illustrate these two modes, a field profile [Fig. 3(c) (right), labeled as G2-2] of G2 is also presented (using same color scale as for G1). We notice that the largest field intensity $\left(\left|E_{x}\right|^{2}\right)$ of the G2 mode is 60 times that of the G1 mode. Furthermore, the leaky energy of G2 mode can penetrate into a large proportion of the fluorescent layer, which is in agreement with the bigger enhancement in the emission spectrum over the region of the G2 mode.

The effective volume of an optical resonant mode is commonly determined by

$$
V=\frac{\int \epsilon_{e}|E|^{2} \mathrm{~d} \mathbf{r}}{\max \left(\epsilon_{e}|E|^{2}\right)}
$$

For plasmons [ [18], the effective energy density [ $\underline{19}] \epsilon_{e}|E|^{2}$ that enters the mode volume is given by $\frac{\epsilon_{0}}{2}\left(n^{2}+\frac{2 n k \omega}{\gamma}\right)|E|^{2}$ using an oscillator model with damping frequency $\gamma$ for the dielectric constants.

We can easily calculate the mode volumes with the field intensity from the 3D-FDTD simulation. The mode volumes for the G1 mode and G2 mode of the photonicplasmonic structure are 0.017 and $0.001 \mu \mathrm{m}^{3}$, respectively. Thus, we can find that the $F_{p}$ of the G2 mode $(\sim 130)$ is about 40 times bigger than that of the G1 mode ( 3.3) in theory. This is because the G2 mode has a much smaller mode volume. In the case presented here, the field confinement in the fluorescent-medium volume offered by the G2 mode allows increased emission with a much bigger enhancement factor. It should be mentioned that the general Purcell factor formula used in our calculations only gives approximate results for photoemission enhancement in plasmonic structures [20].

In conclusion, we have demonstrated a photonicplasmonic structure, consisting of a self-assembled plasmonic substrate and a fluorescent dielectric layer. The structure supports plasmonic modes. The spontaneous emission can be strongly enhanced through coupling to these modes. For the G1 mode, a 3 times enhancement in emission is observed. On the other hand, because of the extremely small mode volume of the G2 mode, the emission is enhanced up to 30 times and the FWHM of the emission spectrum is broadened from 25 to $95 \mathrm{~nm}$. The broadband enhancement is further supported by the reflectance measurement and the FDTD simulation. We believe that the photonic-plasmonic structures fabricated by the simple and economic method can be potentially used in efficient light-emitting devices or broadband luminescent sources.

This work is supported by the 973 Program (grants 2007CB613200 and 2011CB922004), the Catalysis for Sustainable Energy Initiative Center, funded by the Danish Ministry of Science, Technology, and Innovation, and grants 712010 and 10401466 from the Research Grant Council of Hong Kong.

\section{References}

1. R. Ma, R. F. Oulton, V. J. Sorger, G. Bartal, and X. Zhang, Nat. Mater. 10, 110 (2011).

2. T. Suhr, N. Gregersen, M. Lorke, and J. Mørk, Appl. Phys. Lett. 98, 211109 (2011).

3. J. T. Choy, B. J. M. Hausmann, T. M. Babinec, I. Bulu, M. Khan, P. Maletinsky, A. Yacoby, and M. Loncar, Nat. Photon. 5, 738 (2011).

4. K. H. Drexhage, J. Lumin. 1-2, 693 (1970).

5. E. M. Purcell, Phys. Rev. 69, 681 (1946).

6. S. Nakayama, S. Ishida, S. Iwamoto, and Y. Arakawa, Appl. Phys. Lett. 98, 171102 (2011).

7. S. A. Maier, Plasmonics: Fundamentals and Applications (Springer, 2007).

8. M. Kuttge, F. Abajo, and A. Polman, Nano Lett. 10, 1537 (2010).

9. K. Okamato, I. Niki, A. Shvartser, Y. Narukawa, T. Mukai, and A. Scherer, Nat. Mater. 3, 601 (2004).

10. M. C. Tam, H. Su, K. S. Wong, X. Zhu, and H. S. Kwok, Appl. Phys. Lett. 95, 051503 (2009).

11. M. Gwon, E. Lee, D.-W. Kim, K.-J. Yee, M. J. Lee, and Y. S. Kim, Opt. Express 19, 5895 (2011).

12. J. Sun, C. Tang, P. Zhan, Z. Han, Z. Cao, and Z. Wang, Langmuir 26, 7859 (2010).

13. J. C. Bernède, A. Godoy, L. Cattin, F. R. Diaz, M. Morsli, and M. A. del Valle, Solar Energy (INTECH, 2010).

14. T. Tsuzuki and S. Tokito, Adv. Mater. 19, 276 (2007).

15. L. Shi, H. Yin, X. Zhu, X. Liu, and J. Zi, Appl. Phys. Lett. 97, 251111 (2010).

16. X. Zhu, L. Shi, X. Liu, J. Zi, and Z. Wang, Nano Res. 3, 807 (2010).

17. X. Zhu, S. Xiao, L. Shi, X. Liu, J. Zi, O. Hansen, and N. A. Mortensen, Opt. Express 20, 5237 (2012).

18. S. A. Maier, Opt. Express 14, 1957 (2006).

19. R. Ruppin, Phys. Lett. A 299, 309 (2002).

20. A. F. Koenderink, Opt. Lett. 35, 4208 (2010). 